Check for updates

Cite this: RSC Adv., 2018, 8, 2229

\title{
Proinflammatory and osteolysis-inducing effects of 3D printing Ti6Al4V particles in vitro and in vivo
}

Received 22nd November 2017 Accepted 11th December 2017

DOI: $10.1039 / \mathrm{c} 7 \mathrm{ra12677h}$

rsc.li/rsc-advances

\author{
Cuidi Li, (D) $\dagger^{\text {ab }}$ Chuan Jiang, $\dagger^{\mathrm{C}}$ Mingzheng Peng, ${ }^{\mathrm{b}}$ Tao Li, ${ }^{\mathrm{b}}$ Zezheng Yang, ${ }^{\mathrm{b}}$ \\ Zhiyuan Liu, ${ }^{b}$ Ning Li, ${ }^{a}$ Chengtao Wang, ${ }^{a}$ Kerong Dai ${ }^{\star a b}$ and Jinwu Wang ${ }^{\star a b}$
}

\begin{abstract}
Ti6Al4V printing particles have been recently used for fabricating orthopedic implants. Removing these particles completely from fabricated implants is challenging. Furthermore, recycled particles are commonly used in fabrication without additional analysis. Ti6Al4V wear particles derived from orthopedic implants are known to induce inflammatory responses and osteolysis. However, the biosafety of printing particles remains unknown. Here, we investigated the proinflammatory and osteolysisinducing effects of commonly used original and recycled Ti6Al4V printing particles in vitro and in vivo. Our results indicated that although less serious effects were induced compared to wear particles, inflammatory responses and osteoclast-mediated bone resorption were induced by the original printing particles in a particle size-dependent manner. Recycled particles were found to more strongly stimulate bone resorption and inflammatory responses than the original particles; the in vivo effect was enhanced with an increase in particle concentration. Furthermore, the results of our in vitro experiments verified that the printing particles activate macrophages to secrete inflammatory cytokines and promote osteoclastogenesis, which is closely related to particle size and concentration. Taken together, our findings provide a valuable reference for the use of raw printing materials and examination of recycling procedures for implant fabrication.
\end{abstract}

\section{Introduction}

Titanium and Ti6Al4V titanium-alloy biomedical implants have been used widely since the early 1970 s because of their high strength-to-weight ratio, good biocompatibility, and outstanding corrosion resistance. ${ }^{1}$ However, the application of these implants has been hampered by the high cost and poor workability involved in producing complex shapes by using traditional manufacturing processes. For fabrication of surgical implants, 3D printing techniques have recently emerged as a superior choice because they offer design flexibility, costsaving and the ability to fabricate patient-specific shapes featuring extremely high size accuracy. ${ }^{2}$ The two most widely used 3D printing methods are the electron beam melting (EBM) and selective laser melting (SLM) techniques. ${ }^{3-6}$ Porous metal scaffolds fabricated using these techniques have been demonstrated to exhibit high biocompatibility. ${ }^{7-9}$ However, the

${ }^{a}$ School of Biomedical Engineering, Shanghai Jiao Tong University, Shanghai, 200030, China

${ }^{b}$ Shanghai Key Laboratory of Orthopedic Implant, Department of Orthopedic Surgery, Shanghai Ninth People's Hospital Affiliated Shanghai Jiao Tong University School of Medicine, Shanghai 200011, China. E-mail: jinwu_wang@163.com; Fax: +86-2163139920

'Department of Orthopedics, Sun Yat-sen Memorial Hospital, Sun Yat-sen University, Guangzhou, China

$\dagger$ These authors contributed equally to this work. printing particles that are retained deep inside large implants cannot be readily removed completely. ${ }^{10,11}$ Over the past several decades, 1-10 $\mu \mathrm{m}$-sized wear particles generated from metal implants have been reported to elicit inflammatory reactions. ${ }^{12-14}$ Macrophages have been shown to be activated and to release proinflammatory mediators such as tumor necrosis factor-alpha (TNF- $\alpha$ ), interleukin-1 (IL-1), and IL-6. Furthermore, osteoclasts could be activated through a complex sequence of these events, ultimately leading to osteolysis and implant failure..$^{15,16}$

The aforementioned inflammatory responses are widely recognized to be affected by particle size, chemistry, and charge. $^{17,18}$ Because of the different mechanism, spherical particles with different particle sizes, $45-100 \mu \mathrm{m}$ and $20-45 \mu \mathrm{m}$, were used for EBM and SLS techniques. ${ }^{19-21}$ Currently, little is known about the role played by 3D printing particles in these size ranges in inducing inflammation and osteolysis. Furthermore, the residual printing particles from the fabrication platform are commonly recycled and repetitively used after a simple sieving procedure. The biosafety of the recycled printing particles for implant fabrication deserves more attention. Therefore, in this study, we aimed to analyze the in vitro and in vivo inflammatory reaction and osteolysis induced by commonly used Ti6Al4V printing particles featuring distinct particle sizes and by one-time recycled particles, with pure $\sim 2 \mu \mathrm{m}$ Ti particles being used as the positive-control wear particle model. Our 
results could serve as a useful reference when using printing particles or examining the feasibility of recycling procedures for implant fabrication.

\section{Materials and methods}

\subsection{Particle preparation and characterization}

Ti particles, 99.99\% pure, were provided by Johnson Matthey (Ward Hill, MA, USA). The original Ti6Al4V printing particles used here were acquired from three suppliers: EOS $\mathrm{GmbH}$ (Munich, Germany), Waston Medical Appliance Co., Ltd (Changzhou, China), and Arcam AB (Molndal, Sweden). Recycled particles that had undergone one-time recycling and sieving were supplied by Waston Medical Appliance Co., Ltd. These 5 types of particles are referred to in short as Ti, EOS, Waston, Arcam, and Waston recycled particles, respectively. Scanning electron microscopy (SEM, Zeiss LEO 1550) was used to analyze particle morphology; the number average particle sizes was analyzed according to the SEM graphs. Particle size distribution was separately characterized using dynamic laser light scattering (for Ti particles; Zetasizer Nano S, Malvern, Worcestershire, UK) and a laser diffraction particle size analyzer (for printing particles; Mastersizer 2000, Malvern, Worcestershire, UK).

\subsection{In vivo murine calvarial osteolysis model}

We established a murine calvarial osteolysis model as described previously ${ }^{22,23}$ to determine the effects of particles on osteolysis in vivo. In total, 54 healthy male C57BL/6 mice (8 weeks old, 20$23 \mathrm{~g}$ ) were obtained from SLAC Laboratory Animal Co., Ltd. (Shanghai, China). All the animal experiments were performed in accordance with the guidelines and regulations for the care and use of laboratory animals of the National Institutes of Health. All procedures were approved by the Institutional Animal Care and Use Committee of Shanghai Jiao Tong University School of Medicine. Briefly, mice were anesthetized by intraperitoneal injection of $50 \mathrm{mg} \mathrm{kg}{ }^{-1}$ pentobarbital, and then the periosteum was separated from the calvarium by using a surgical scalpel. Particles were dispersed in saline at a low concentration $(10 \mathrm{mg})$ or high concentration $(30 \mathrm{mg})$ and injected on the surfaces of the bilateral parietal bones. In the sham control, the incision was closed without further intervention. After 14 days, mice were sacrificed for further analysis.

\section{3. $\mu \mathrm{CT}$ imaging}

Calvarium samples were analyzed by using a $\mu \mathrm{CT}$ system ( $\mu \mathrm{CT}$ 80; SCANCO Medical AG, Bassersdorf, Switzerland) at an isometric resolution of $9 \mathrm{~mm}$ and X-ray energy settings of $70 \mathrm{kV}$, $114 \mu \mathrm{A}$, and $8 \mathrm{~W}$; $3 \mathrm{D}$ reconstructed images were obtained using the manufacturer's software. The number of pores and the porosity in a square-shaped region of interest $(3.5 \mathrm{~mm} \times 3.5$ $\mathrm{mm}$ ) were analyzed using Image-Pro Plus 6.0 software.

\subsection{Histological staining and histomorphometric analysis}

The collected calvaria were fixed with $4 \%$ paraformaldehyde (PFA) for 3 days, decalcified in 10\% EDTA for 1 month, and embedded in paraffin. Cross-sections $(5 \mathrm{~mm})$ were cut in the coronal plane on a microtome. Afterwards, the sections were performed with hematoxylin/eosin (HE) and tartrate-resistant acid phosphatase (TRAP) staining and observed under an optical microscope (Leica DM4000B). The bone area and osteoclast number were calculated using Image-Pro Plus 6.0 software.

\subsection{Enzyme-linked immunosorbent assay (ELISA) for analyzing inflammation in vivo}

As previously described, ${ }^{24,25}$ each of the harvested calvaria was carefully placed into one well of a 12-well plate and incubated at $37^{\circ} \mathrm{C}$ and $5 \% \mathrm{CO}_{2}$ in $1 \mathrm{~mL}$ of serum- and phenol-free Dulbecco's modified Eagle medium (DMEM; Invitrogen, Carlsbad, CA, USA) with $100 \mathrm{U} \mathrm{mL}^{-1}$ penicillin and $100 \mathrm{U} \mathrm{mL}^{-1}$ streptomycin. After $24 \mathrm{~h}$ of culture, the culture medium was collected for analysis of TNF- $\alpha$ and IL-6 levels using the respective ELISA kits purchased from R \& D Systems (Minneapolis, MN, USA). Assays were performed according to the manufacturer's instructions.

\subsection{Proinflammatory assay in vitro}

2.6.1. Cell culture and viability. Murine macrophage/ monocyte cell line RAW264.7 cells (Shanghai Institute of Cellular Biology; Chinese Academy of Sciences, Shanghai, China) were maintained at $37{ }^{\circ} \mathrm{C}$ and $5 \% \mathrm{CO}_{2}$ in $\alpha$-minimum essential medium ( $\alpha$-MEM; Invitrogen, Carlsbad, CA, USA) containing $10 \%$ fetal bovine serum (FBS; Gibco, Gaithersburg, $\mathrm{MD}, \mathrm{USA}), 100 \mathrm{U} \mathrm{mL}^{-1}$ penicillin, and $100 \mathrm{U} \mathrm{mL}^{-1}$ streptomycin. The effects of particle size and concentration on RAW264.7 cell viability were investigated using the cell counting kit-8 (CCK-8; Dojindo Molecular Technology, Inc., Kumamoto, Japan) assay. Cells $\left(1 \times 10^{4}\right.$ per well $)$ were plated in 96-well plates in triplicate and incubated for $2 \mathrm{~h}$. After the cells were stably attached to the plates, the culture medium was replaced with medium in which different particles were dispersed at concentrations of $0,1,5,10,20,40$, and $80 \mathrm{mg} \mathrm{mL}^{-1}$; the cells were exposed to the particles for 1 or 3 days. Subsequently, cell viability was analyzed by performing the CCK-8 assay according to the manufacturer's instructions. Cells cultured with standard media were used as the controls. Results are reported as the percentage ratio: $\mathrm{OD}_{\text {sample }} / \mathrm{OD}_{\text {control }} \times 100 \%(n=3)$.

2.6.2. Cell morphology. RAW 264.7 cells $\left(5 \times 10^{4}\right.$ per well $)$ were plated in 24-well plates in triplicate and incubated for $2 \mathrm{~h}$. After the cells were stably attached to the plates, the medium was replaced with medium in which $10 \mathrm{mg} \mathrm{mL}^{-1} \mathrm{Ti}$, Waston, Waston recycled, EOS, or Arcam particles was dispersed. The effects of particle size on the morphology of RAW264.7 cells were observed after 3 days of culture. First, cells were washed with phosphate buffer solution and fixed with $4 \%$ PFA. On one hand, cells on and around the particles were observed using SEM (Quanta 250, FEI, Hillsboro, OR, USA) after gradient elution with ethanol and thermostatic drying; On the other hand, the cell nuclei were stained with DAPI (Sigma-Aldrich, St. Louis, MO, USA) and observed using confocal laser-scanning microscopy (CLSM; Nikon, Tokyo, Japan). 


\subsection{ELISA for in vitro analyses}

RAW264.7 cells $\left(5 \times 10^{4}\right.$ per well) were plated in 24 -well plates in triplicate and incubated for $2 \mathrm{~h}$. After the cells were attached to the plates, the medium was replaced with the dispersion liquid containing $10 \mathrm{mg} \mathrm{mL}{ }^{-1} \mathrm{Ti}$, Waston, Waston recycled, EOS, or Arcam particles. After culturing for 1 or 3 days, the culture media were collected and the TNF- $\alpha$ and IL- 6 concentrations were determined using the ELISA kit from $\mathrm{R}$ \& $\mathrm{D}$ Systems (see Section 2.5). As the control, medium was collected from cells cultured in parallel in the absence of particles. Each experiment was performed three times in triplicate.

\subsection{Osteoclastogenesis assay in vitro}

2.8.1. Preparation and culture of mouse bone marrowderived monocytes. To examine the effect of different printing particles on osteoclastogenesis in vitro, mouse bone marrowderived monocytes (BMMs) were isolated as previously described. ${ }^{26,27}$ All the animal experiments were performed in accordance with the guidelines and regulations for the care and use of laboratory animals of the National Institutes of Health. All procedures were approved by the Institutional Animal Care and Use Committee of Shanghai Jiao Tong University School of Medicine. Briefly, bone marrow cells collected from tibiae and femurs were incubated in a T75 flask in $\alpha$-MEM containing $10 \%$ (v/v) FBS and $30 \mathrm{ng} \mathrm{mL}^{-1}$ macrophage colony-stimulating factor (M-CSF; PeproTech, Rocky Hill, NJ, USA) at $37{ }^{\circ} \mathrm{C}$ in a humidified atmosphere of $5 \% \mathrm{CO}_{2}$ and $95 \%$ air. After incubation for 3 days, the medium was changed to remove non-adherent cells and impurities, and the adherent cells were used as BMMs. The incubation was continued for an additional 4 days. After reaching $90 \%$ confluence, the BMMs were harvested through trypsin digestion and seeded into culture plates for further experiments.

2.8.2. Cell viability. The effects of particle size and concentration on the viability of BMMs were investigated using the CCK- 8 assay. BMMs $\left(1 \times 10^{4}\right.$ per well $)$ were plated in 96-well plates in triplicate and incubated for $2 \mathrm{~h}$. After cells were stably attached to the plates, the medium was replaced with the dispersion liquid containing different particles at concentrations of $0,1,5,10,20,40$, and $80 \mathrm{mg} \mathrm{mL}^{-1}$. After the cells were cultured with the particles for 1,3 , or 5 days, cell viability was analyzed by CCK-8 assay according to the manufacturer's instructions. Cells cultured with standard media were used as the control. Results are reported as the percentage ratio: $\mathrm{OD}_{\text {sample }} / \mathrm{OD}_{\text {control }} \times 100 \%(n=3)$.

2.8.3. Osteoclastogenesis induced with different particles. Based on the CCK-8 results, the osteoclastogenic effects of different particles on BMMs were subsequently assayed. BMMs $\left(8 \times 10^{3}\right.$ per well $)$ were plated and incubated in 96-well plates in triplicate. After $24 \mathrm{~h}$ of culture, the medium was replaced with fresh osteoclast differentiation medium containing $30 \mathrm{ng} \mathrm{mL} \mathrm{m}^{-1}$ M-CSF, $100 \mathrm{ng} \mathrm{mL}^{-1}$ Receptor Activator for Nuclear Factor- $\kappa \mathrm{B}$ Ligand (RANKL; PeproTech), and different types of particles ( $1 \mathrm{mg} \mathrm{mL} \mathrm{mL}^{-1}$ in culture medium). The culture medium was changed every $48 \mathrm{~h}$ carefully without removing the particles. For the control, cells were cultured in the osteoclast differentiation medium without any added particles. After 3 or 5 days of culture, cells were immobilized and stained using a TRAP staining kit (387A-1KT, Sigma-Aldrich); TRAP-positive cells containing more than three nuclei were regarded as multinucleated osteoclasts. Osteoclasts were counted under an optical microscope. The TRAP-positive multinucleated cell area was measured using Image-Pro Plus.

\subsection{Statistical analysis}

SPSS 17.0 software (SPSS, Inc., Chicago, IL, USA) was used to analyze the data obtained from at least three independent experiments. Data are expressed as the mean \pm standard deviation (SD). Statistical analysis was performed using one-way analysis of variance (ANOVA). $P<0.05$ was considered statistically significant.

\section{Results and discussion}

\subsection{Characterization of different particles}

Morphology and particle-size distributions of different particles are shown in Fig. 1. The SEM results (Fig. 1A) revealed that Ti particles featured irregular shapes as expected for a typical wear particle model, whereas the particles used for 3D printing systems featured a regular spherical shape with a smooth surface. The results also showed clear differences in particle sizes; according to our calculations (Fig. 1B and Table 1), Ti particles $(2.62 \pm 1.25 \mu \mathrm{m})$ showed a typical size around $2 \mu \mathrm{m}$. Arcam particles presented the largest particle size (58.93 \pm $7.59 \mu \mathrm{m})$ among the printing particles, EOS particles featured a relatively intermediate size $(35.44 \pm 3.96 \mu \mathrm{m})$, whereas Waston $(26.15 \pm 4.59 \mu \mathrm{m})$ and Waston recycled particles $(23.97$

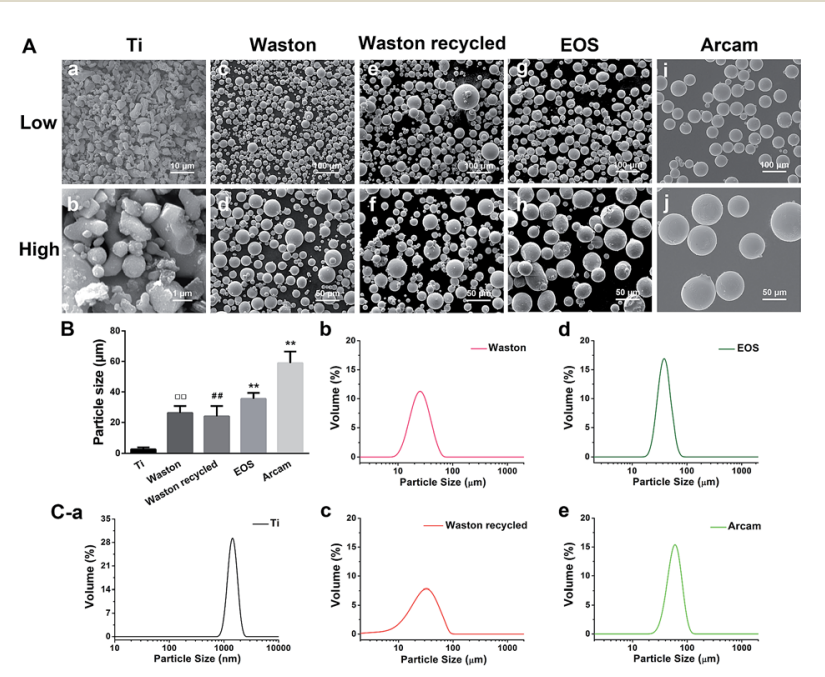

Fig. 1 (A) SEM images of (a) $5000 \times$ and (b) $40000 \times$ Ti particles; (c) $500 \times$ and (d) $1000 \times$ Waston particles; (e) $500 \times$ and (f) $1000 \times$ Waston recycled particles; (g) $500 \times$ and (h) $1000 \times$ EOS particles; and (i) $500 \times$ and (j) $1000 \times$ Arcam particles. (B) Number average particle sizes according to SEM images. (\#\#P<0.01 vs. Ti group; $O \bigcirc P<0.01$ vs. Waston group; is 2 is 0.01 vs. EOS group). (C) Laser-particle analysis of the cumulative distribution (continuous line) and mean volume particle sizes of (a) Ti, (b) Waston, (c) Waston recycled, (d) EOS, and (e) Arcam particles. 
Table 1 The calculated size of different particles

\begin{tabular}{lcc}
\hline Particles & Mean size $(\mu \mathrm{m})$ & Surface area $\left(\mathrm{cm}^{2} \mathrm{mg}^{-1}\right)$ \\
\hline Ti & $2.62 \pm 1.25$ & 5.20 \\
Waston & $26.15 \pm 4.59$ & 0.52 \\
Waston recycled & $23.97 \pm 6.78$ & 0.56 \\
EOS & $35.44 \pm 3.96$ & 0.38 \\
Arcam & $58.93 \pm 7.59$ & 0.23
\end{tabular}

$\pm 6.78 \mu \mathrm{m}$ ) presented the smallest size. Moreover, the Waston recycled particles contained a higher number of smaller and larger particles than the original particles, which might be due to the lasing and recycling process. We further analyzed the size distribution of the particles by using the laser diffraction method (Fig. 1C). The Ti, EOS, and Arcam particles exhibited a relatively uniform size distribution. Among all of the examined particles, Ti particles presented the smallest mean volume particle size and Arcam particles presented the largest size. The Waston particles (Fig. 1C-b) and Waston recycled particles (Fig. 1C-c) featured smaller mean volume particle sizes than other printing particles, but their size distribution, particularly that of Waston recycled particles, was wider than that of EOS and Arcam particles, which supported our imaging results.

\subsection{Effects of particles on osteoclast-mediated bone resorption in vivo}

3.2.1. $\quad \mu$ CT assay. Osteolysis induced by the low and high concentrations of the particles was first studied using $\mu \mathrm{CT}$ analysis (Fig. 2). The 3D reconstructed $\mu \mathrm{CT}$ images of the calvarial bone (Fig. 2A) and quantification of the results (Fig. 2B) revealed that exposure to the particles induced distinct extents of osteolysis (reflected by the number of pores and the percentage of porosity on the bone surface) relative to sham treatment $(P<0.01)$. However, bone destruction induced by the printing particles was considerably less serious than that induced by the positive-control Ti particles $(P<0.01)$. Among the original printing particles, Waston particles, which were the smallest, triggered the most serious bone destruction, whereas EOS particles, which were of an intermediate size, produced the weakest effect. Unexpectedly, we also found that recycled Waston particles caused more serious bone destruction as compared to the original particles. Furthermore, our results showed that bone loss could be induced by the printing particles sensitively. Even at a relatively low concentration, the
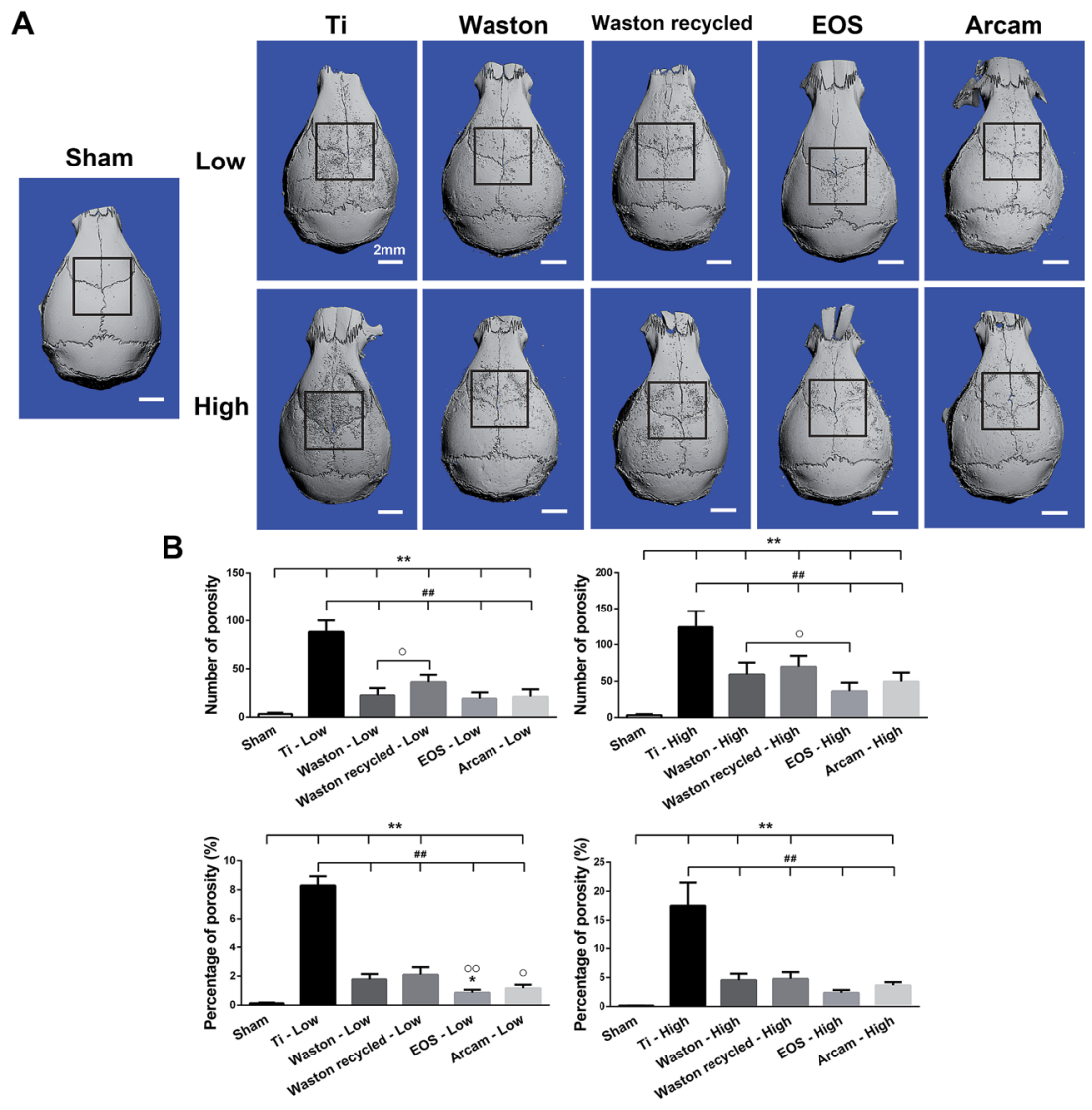

Fig. 2 (A) Representative 3D reconstructed $\mu \mathrm{CT}$ images from each group. (B) Number of pores and percentage of total porosity of each sample $(* P<0.05$ and $* * P<0.01$ vs. Sham; \#\#P<0.01 vs. Ti group; $O P<0.05$ and $\bigcirc \bigcirc P<0.01$ vs. Waston group). 
particles exerted the same size-dependent effect on bone destruction.

\subsubsection{Histological staining and histomorphometric analysis}

3.2.2.1. Histological staining analysis. To further verify the osteolysis induced by the particles, we performed HE staining (Fig. 3). The results confirmed the aforementioned phenomenon observed using $\mu \mathrm{CT}$ imaging, with regard to the different extents of bone destruction induced by the particles. When the printing particles were implanted at a low concentration, osteolysis was induced weakly inside the bone tissue; by contrast, at a high concentration, all particles triggered serious osteolysis inside the bone tissue as compared to sham treatment (bone area $\left.=0.2501 \pm 0.0308 \mathrm{~mm}^{2}\right)(P<0.01)$. The bone area measured for the Ti group $\left(0.1375 \pm 0.0406 \mathrm{~mm}^{2}\right)$ was significantly smaller than that measured for the other particle groups $(P<0.01)$, whereas the area was considerably larger for the EOS group $\left(0.1837 \pm 0.0432 \mathrm{~mm}^{2}\right)$; the Waston recycled group $\left(0.1537 \pm 0.0477 \mathrm{~mm}^{2}\right)$ showed the second smallest bone area, followed by the Waston group $(0.1621 \pm$ $0.0299 \mathrm{~mm}^{2}$ ).

3.2.2.2. Histomorphometric analysis. Osteoclast formation is known to be responsible for both physiological and pathological bone resorption. ${ }^{28}$ Therefore, we identified the osteoclasts present in bone tissue by TRAP staining (Fig. 4). Although bone loss was induced weakly, TRAP-positive osteoclasts were sensitively induced by all the printing particles while implanted at low concentration. After implantation of particles at high concentration, abundant osteoclasts were observed along the eroded bone surface inside the calvarial bone. The trend observed in the particle-induced TRAP- positive multinucleated osteoclasts was consistent with bone-loss results.

3.2.3. ELISA analysis of proinflammatory cytokines in vivo. To investigate the mechanisms underlying the effects of particles on osteolysis, we measured levels of the proinflammatory cytokines TNF- $\alpha$ and IL- 6 by using ELISA (Fig. 5). TNF- $\alpha$ levels induced by the particles were significantly higher than those in the sham group $(P<0.01)$. All printing particles induced lower levels of TNF- $\alpha$ than the Ti particles at both low and high concentrations. Although no significant difference was observed among the printing particles, Waston and Waston recycled particles showed a stronger stimulation effect.

The tested particles also induced higher levels of IL- 6 as compared to the level in the sham group $(P<0.01)$. When implanted at a low concentration, all particles induced IL-6 at low levels, and levels induced by printing particles did not differ markedly from those induced by positive-control Ti particles. However, at a high concentration, the levels of IL-6 induced by the printing particles were significantly lower than that induced by the $\mathrm{Ti}$ particles $(P<0.01)$. Among the original printing particles, Waston particles most strongly stimulated IL-6 production $(P<0.01)$. Additionally, Waston recycled particles resulted in a considerable increase in IL-6 production relative to that induced by the original particles $(P<0.01)$.

\subsection{Proinflammatory assay in vitro}

3.3.1. Cell viability and morphology. The effects of particle size and concentration on the viability of macrophages RAW264.7 were determined by CCK-8 assays after 1 and 3 days of culture (Fig. 6). Printing particles did not appear to exhibit cytotoxicity toward macrophages; cell viability was high after

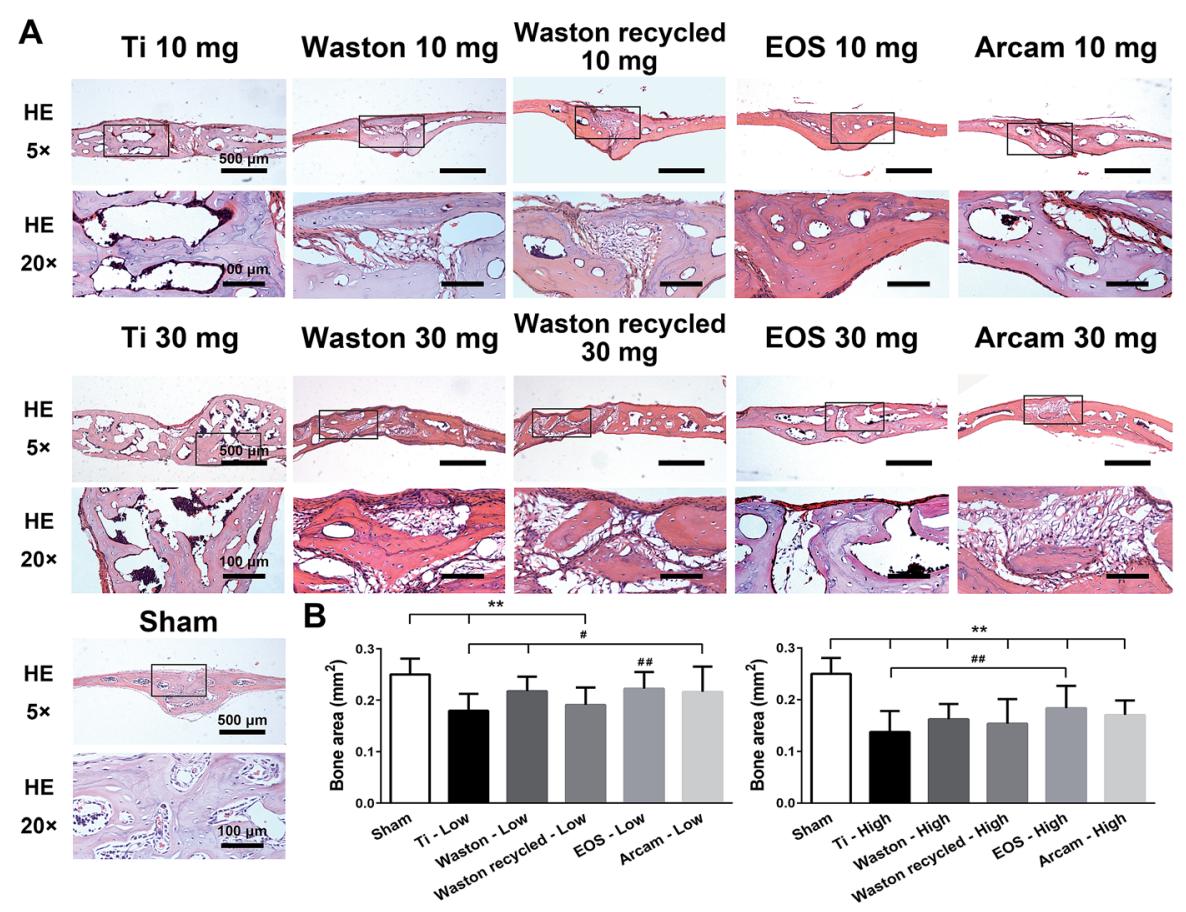

Fig. 3 Histological staining of calvarial sections. (A) HE staining $(\times 5, \times 20)$ after 14 days of implantation of particles at low and high concentrations. (B) Quantification of bone area $\left(\mathrm{mm}^{2}\right)$ in each group (**P<0.01 vs. Sham; \#P<0.05 and \#\#P<0.01 vs. Ti group). 

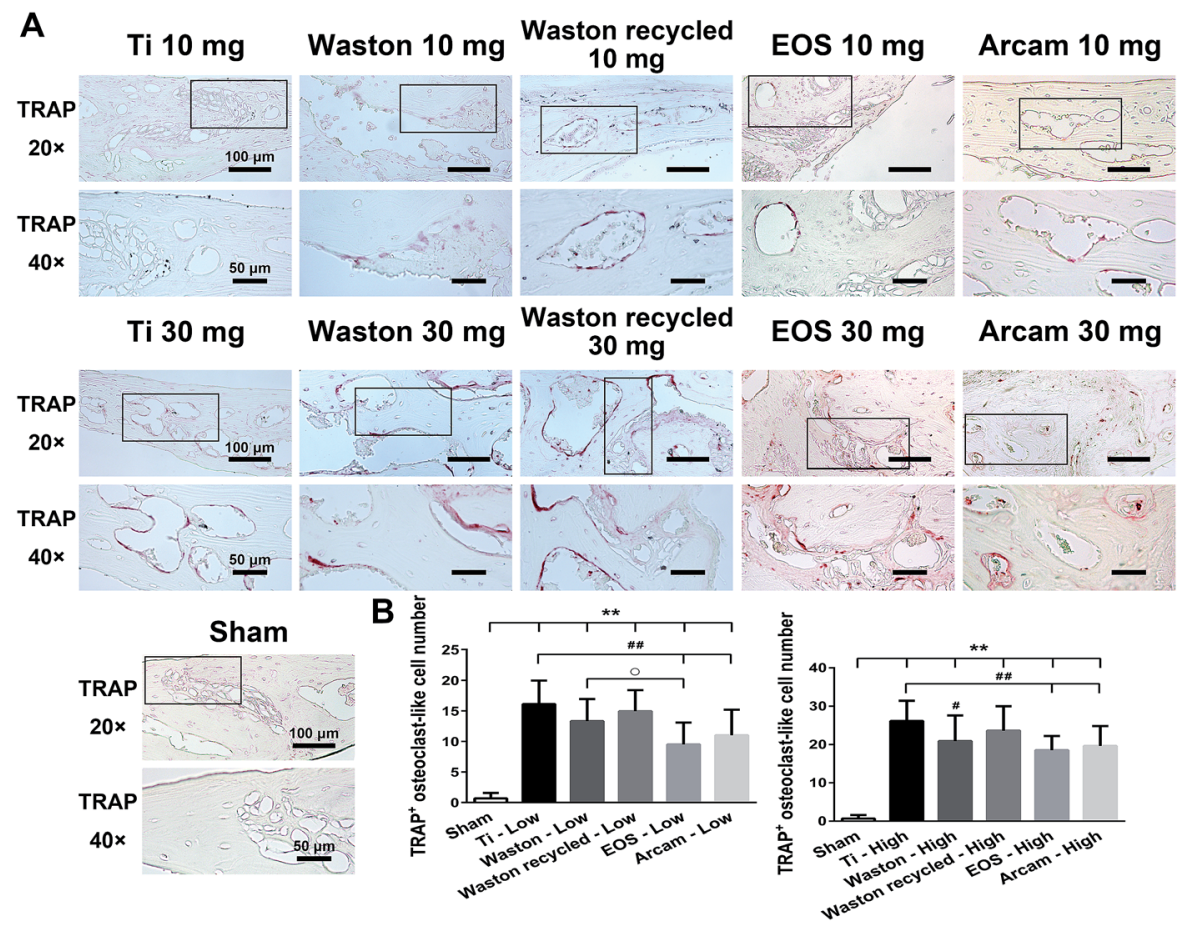

Fig. 4 Histomorphometric analysis of calvarial sections. (A) TRAP staining $(\times 5, \times 20)$ after 14 days of implantation of particles at low and high concentrations. (B) Quantification of TRAP-positive multinucleated osteoclasts in each group $(* * P<0.01 \mathrm{vs}$. Sham; \#P<0.05 and \#\#P<0.01 vs. Ti group; $O P<0.05$ vs. Waston group).

exposure to the particles at each concentration and time point. However, Ti particles induced high cytotoxicity at concentrations higher than $10 \mathrm{mg} \mathrm{mL}^{-1}$. Therefore, cell morphology after 3 days of culture with the particles at $10 \mathrm{mg} \mathrm{mL}^{-1}$ was analyzed using SEM and CLSM (Fig. 7). The SEM images from the top view (Fig. 7A) and CLSM images from the bottom view (Fig. 7B) revealed that the cells grew around EOS and Arcam particles, whereas they mostly grew under Ti particles. Notably, numerous cells were observed to grow on the Waston and Waston recycled particles and encompass most of them in the SEM images. CLSM images from the side view (Fig. 7C) further verified that more
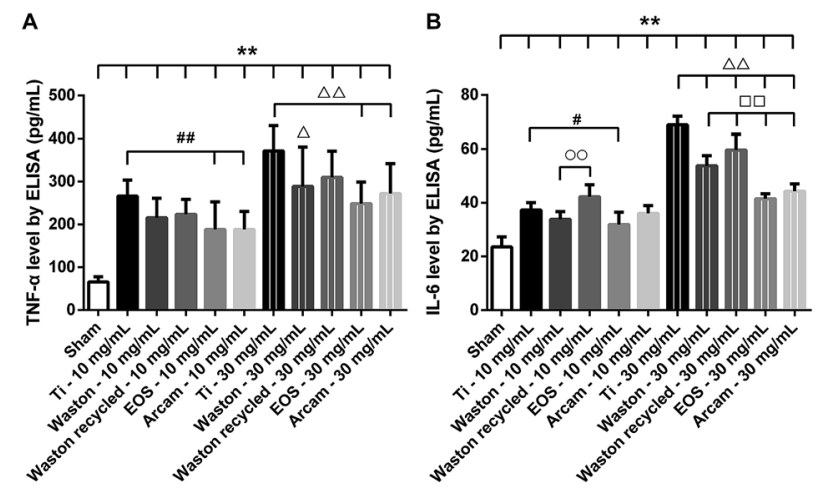

Fig. 5 Effects of particle size and particle concentration on proinflammatory cytokines in vivo. Levels (pg mL ${ }^{-1}$ ) of (A) TNF- $\alpha$ and (B) IL-6 $\left(* * P<0.01\right.$ vs. Sham; $\# P<0.05$ and $\# \# P<0.01$ vs. $10 \mathrm{mg} \mathrm{mL}^{-1} \mathrm{Ti}$ group; $O P<0.05$ and $\bigcirc \bigcirc P<0.01$ vs. $10 \mathrm{mg} \mathrm{mL}^{-1}$ Waston group; $\Delta P$ $<0.05$ and $\Delta \Delta P<0.01$ vs. $30 \mathrm{mg} \mathrm{mL}^{-1}$ Ti group; $\square \square P<0.05$ vs. $30 \mathrm{mg}$ $\mathrm{mL}^{-1}$ Waston group). cells harboring an intact nucleus grew on the Waston and Waston recycled particles than on Ti, EOS, and Arcam particles.

3.3.2. ELISA analysis in vitro. To quantify the inflammatory response of macrophages to the particles, we measured the levels of TNF- $\alpha$ and IL-6 produced by RAW264.7 cells after 1 and 3 days of culture. After 1 day of culture, EOS and Arcam particles exerted no effect on TNF- $\alpha$ production relative to control

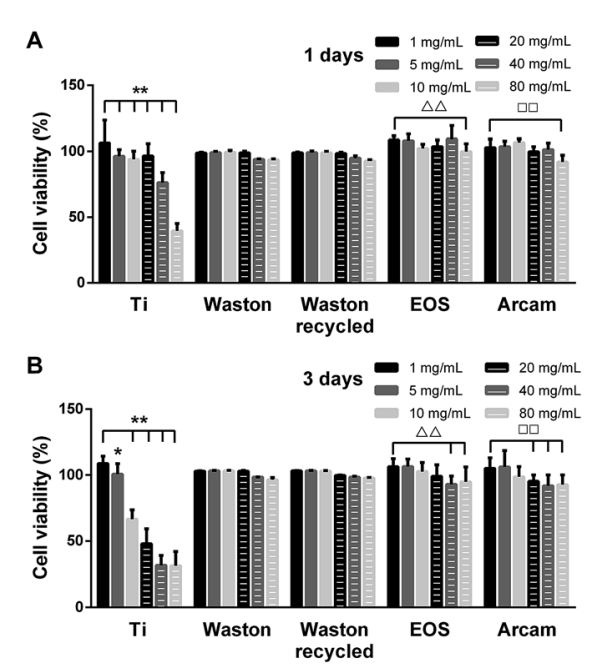

Fig. 6 Effects of particle size and particle concentration on RAW264.7 cell viability. CCK- 8 assay of the proliferation of cells cultured with different concentrations of various particles for 1 and 3 days. (Ti group: $* P<0.05$ and $* * P<0.01 \mathrm{vs.} 1 \mathrm{mg} \mathrm{mL}^{-1}$ particles; EOS group: $\Delta \Delta P<$ 0.01 vs. $1 \mathrm{mg} \mathrm{mL}^{-1}$ particles; Arcam group: $\square \square P<0.05$ vs. $1 \mathrm{mg} \mathrm{mL}^{-1}$ particles). 


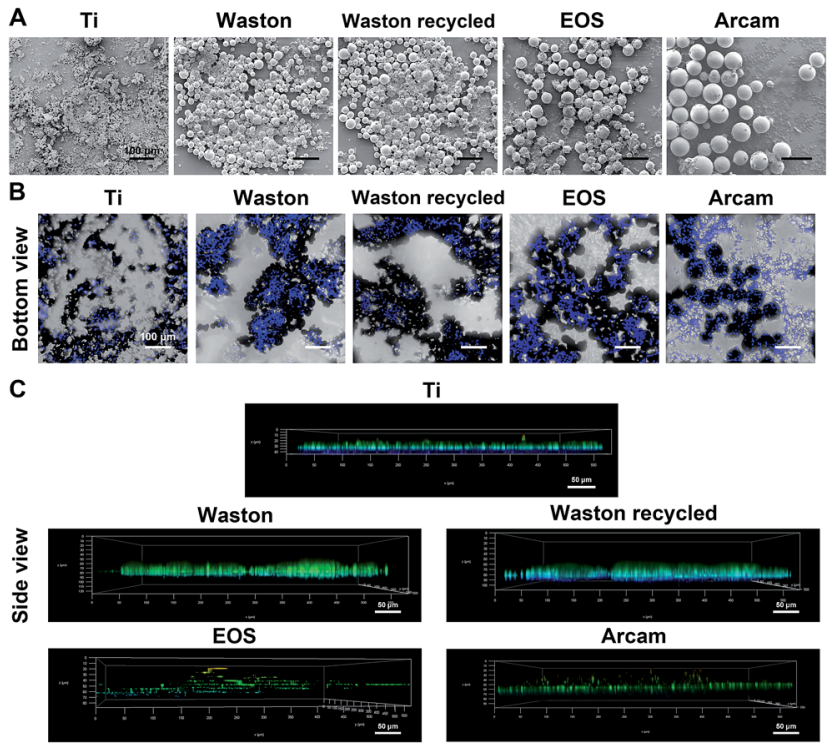

Fig. 7 Effects of particle size and particle concentration on RAW264.7 cell growth and morphology. Growth and morphology of cells cultured with various particles after 3 days of culture. (A) SEM images from top view; (B, C) CLSM images of cells with DAPI-stained nuclei (blue) from bottom view and using a deep-related mode from a side view.

(Fig. 8A). However, all tested particles induced higher levels of TNF- $\alpha$ and IL- 6 production relative to the control after 3 days of culture $(P<0.01)$. The levels of TNF- $\alpha$ and IL- 6 induced by the
A

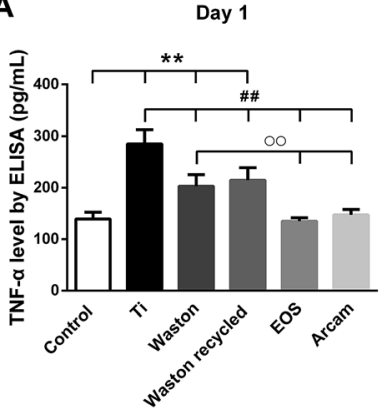

B

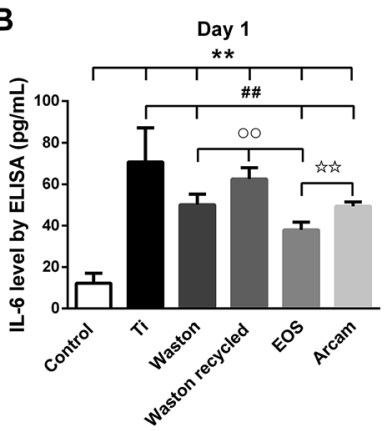

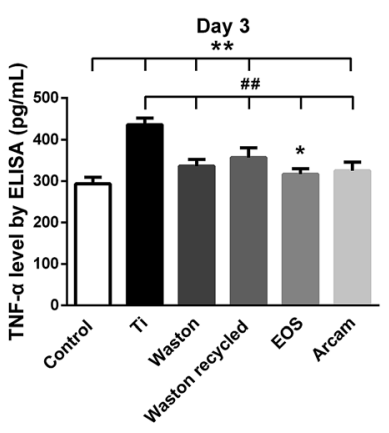

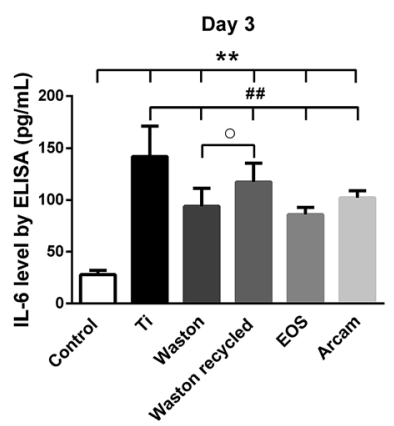

Fig. 8 Effects of particle size and particle concentration on proinflammatory cytokines in vitro. (A) TNF- $\alpha$ and (B) IL- 6 production by RAW264.7 cells after 1 and 3 days of culture with particles at $10 \mathrm{mg}$ $\mathrm{mL}^{-1}\left(* P<0.05\right.$ and $* * P<0.01$ vs. Sham; \#\#P<0.01 vs. $10 \mathrm{mg} \mathrm{mL}^{-1} \mathrm{Ti}$ group; $O P<0.05$ and $\bigcirc \bigcirc P<0.01$ vs. $10 \mathrm{mg} \mathrm{mL}^{-1}$ Waston group; 论许 $P<0.01$ vs. $10 \mathrm{mg} \mathrm{mL}^{-1}$ EOS group). printing particles were considerably lower than those induced by Ti particles. Although the levels of the proinflammatory cytokines induced by the original printing particles did not differ significantly, EOS particles showed the weakest induction of proinflammatory cytokine secretion; and Waston recycled particles induced higher levels of IL-6 production than the original particles $(P<0.05)$.

\subsection{Osteoclastogenesis assay in vitro}

3.4.1. Cell viability. In contrast to RAW264.7 cells, BMMs were highly sensitive to the effects of particle concentration and size (Fig. 9A). Whereas cell proliferation was strongly inhibited during 5 days of culture with Ti, Waston, and Waston recycled particles, the cells grew well when cultured with EOS and Arcam particles. Furthermore, the inhibitory effect increased markedly with the concentration of the particles.

3.4.2. Osteoclastogenesis induced by distinct particles. Based on the results of the cell viability assays and the operability of the particles, the stimulation of osteoclast formation by various particles applied at $1 \mathrm{mg} \mathrm{mL}^{-1}$ was studied. BMMs were cultured with the particles for 3-5 days. Subsequently, TRAPpositive multinucleated cells were stained and analyzed (Fig. 9B and C). Most of the printing particles induced an increase in the number and area of TRAP-positive multinucleated osteoclasts during the culture period. After 5 days of culture, the following trend was observed: Waston and Waston recycled particles most strongly induced osteoclast formation, whereas the number and area of osteoclasts after culture with EOS particles were similar to those after culture in the absence of added particles. Few osteoclasts were observed in the culture plate of Ti particles during the entire culture period. This might be due to the drastic decrease in cell viability; because the cells were dead or dying, they were probably incapable of fusing into osteoclasts. The difference between the results obtained in the in vitro and in vivo experiments might be due to the release of cytokines by the dying cells in vivo, which could attract fresh macrophages from nearby tissues or the blood circulation.

\section{Discussion}

Clinical use of 3D-printed titanium-alloy orthopedic implants has been gradually increasing. The printing particles retained inside the implants are hard to be removed completely. To the best of our knowledge, no previous study has investigated the biological activity of printing particles sized between 20 and 100 $\mu \mathrm{m}$ at cell and tissue level, including the inflammatory responses and osteolysis elicited by these particles. In addition, particles are usually recycled and used after a simple sieving process. Whether differences of biocompatibility exist between the recycled particles and original particles remains unknown. The chemical composition, size, charge, and surface area of these particles are believed to primarily determine the intensity of the foreign body macrophage response and the extent of osteolysis. ${ }^{17,18,29}$ With particles possessing uniform composition and spherical morphology, we examined how particle size, particle concentration, and recycling of the particles affected 


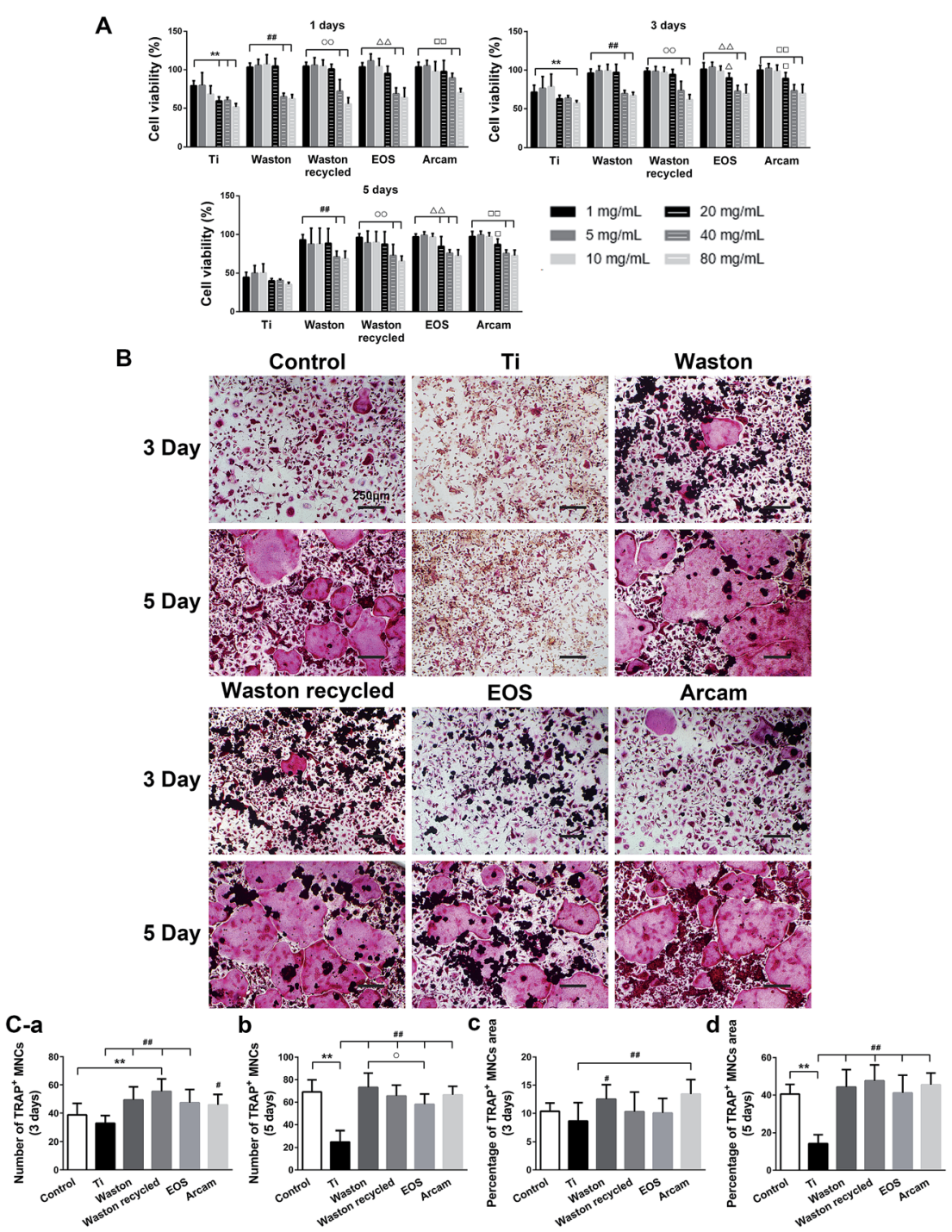

Fig. 9 Effects of particle size and particle concentration on osteoclastogenesis in vitro. (A) Proliferation of BMMs cultured with particles at different concentrations for 1, 3, and 5 days, measured using the CCK-8 assay (Ti group: ** $P<0.01 \mathrm{vs.} 1 \mathrm{mg} \mathrm{mL}^{-1}$ particles; Waston group: \#\#P< $0.01 \mathrm{vs} .1 \mathrm{mg} \mathrm{mL}^{-1}$ particles; Waston recycled group: $\bigcirc \bigcirc P<0.01 \mathrm{vs} .1 \mathrm{mg} \mathrm{mL}^{-1}$ particles; EOS group: $\Delta P<0.05 \mathrm{and} \Delta \Delta P<0.01 \mathrm{vs} .1 \mathrm{mg} \mathrm{mL}^{-1}$ particles; Arcam group: $\square P<0.05$ and $\square \square P<0.01$ vs. $1 \mathrm{mg} \mathrm{mL}^{-1}$ particles). (B) Osteoclasts differentiated from BMMs after being cultured with $1 \mathrm{mg} \mathrm{mL}^{-1}$ particles for 3 and 5 days were identified through TRAP staining $(\times 10)$. (C) Quantification of TRAP-positive multinucleated cells and their area percentage (**P< 0.01 vs. Sham; $\# P<0.05$ and \#\#P<0.01 vs. Ti group; $O P<0.05$ vs. Waston group).

inflammation/osteolysis-stimulating and osteoclastogenesis in vitro and in vivo with the classic wear particle model (Ti particles) used as the positive control.

First, our physical characterizations verified the specific differences of particle sizes used for EBM and SLM as reported previously. ${ }^{19-21}$ Although the particles were used with the same printing technique, Waston particles were found to feature smaller mean particle sizes than EOS particles. Moreover, although the composition was unchanged (data not shown), Waston recycled particles displayed a wider particle size distribution as compared to the original particles.

To study the biological activity of the printing particles in vivo, an immunocompetent $\mathrm{C} 57 \mathrm{BL} / 6$ murine model that produces foreign-body responses similar to those observed in human patients was used. ${ }^{30}$ Results of histological staining and $3 \mathrm{D}$ reconstructed $\mu \mathrm{CT}$ images verified that the particle size and concentration affected bone destruction on both the surface and inside of the calvarial bone. The severity of the effects observed in the groups showed the following trend: Sham $<$ EOS $<$ Arcam $<$ Waston $<$ Waston recycled $<$ Ti. Printing particles that around $20 \mu \mathrm{m}$ or $60 \mu \mathrm{m}$ might exert strong osteolysis-inducing effects. Recycled Waston particles caused more serious osteolysis than the original particles, which might be owing to the recycled particles including a higher number of small and large particles when compared to the original particles, as was clearly observed in the SEM images. Analysis of TRAP-positive osteoclast formation in the bone destruction area revealed a similar trend of particle size- and concentration-dependent effects and confirmed our hypothesis that the osteolysis-induction effect of the printing particles is closely related to osteoclast activation.

Inflammatory responses have been implicated as a major contributor to increased osteoclastic activity and accelerated 
osteolysis. ${ }^{15,16,31,32}$ Titanium-alloy wear particles $(0.5-10 \mu \mathrm{m})$ are widely recognized to play a critical role in osteolysis by inducing inflammatory responses. ${ }^{33-35}$ Recently, large particles at the macro level (size of $>100 \mu \mathrm{m}$ ) have been observed to lead to a maintenance of foreign-body reaction and inflammatory responses. $^{36-38}$ Thus, we hypothesis that the inflammatory response might be an underlying reason for the size-dependent osteoclast-activating and osteolysis-inducing effects of printing particles observed here.

Moreover, an interesting phenomenon was also observed here: serious osteolysis inside the bone tissue was observed in the histological analysis, whereas the bone loss induced on the surface by the printing particles appeared to be comparatively weak according to the imaging results. This phenomenon further supports our inflammatory response hypothesis that the particles activate macrophages as wear particles to secrete proinflammatory cytokines, including IL-1, IL-6, and TNF- $\alpha$, which indirectly stimulate osteoclastic bone resorption and promote the recruitment of mononuclear cells into these osteolytic lesions. ${ }^{15,16,39-43}$

Given these findings, we measured the concentration of the proinflammatory cytokines, IL- 6 and TNF- $\alpha$, in the medium of cultured calvaria. The levels of both cytokines were increased drastically after implantation of the particles at either a low or high concentration. Furthermore, the severity of the effect measured here showed a similar trend as that observed in the osteolysis results, which implies that osteolysis is related to the inflammatory response and the secretion of the proinflammatory cytokines, TNF- $\alpha$ and IL- 6 .

To further study how the particles triggered inflammation and osteoclastogenesis in vitro, the model cells for monocytes and macrophages, BMMs and RAW264.7 cells, respectively, were used. Being different from Ti group which showed cytotoxicity, the printing particles were found to be nontoxic to the RAW264.7 cells. In addition, much more cells were observed around and on the original and recycled Waston particles than other printing particles in the SEM and CLSM results (Fig. 7). Furthermore, the levels of the cytokines TNF- $\alpha$ and IL-6 increased with time and the effect showed a size-dependence similar to that observed in the osteolysis assay in vivo. Thus, we hypothesized that the size-dependent induction effects of the particles might be partly relevant to their role in cell accumulation and attachment. It is known that wear particles swiftly adsorb a layer of host proteins immediately after implantation and exposure to physiologic fluids. ${ }^{44}$ Upon binding to the proteins adsorbed on biomaterial surfaces, macrophages that are recruited to the implant site could be activated, resulting in release of inflammatory cytokines such as TNF- $\alpha$, IL-6, and IL-1 ${ }^{\text {44-46 }}$ and accelerated osteolysis. ${ }^{39-43,47,48}$ Among the printing particles, Waston original and recycled particles, with a smaller particle size and larger specific surface area per unit weight (Table 1), contacted with more cells and thereby probably led to the more pro-inflammation cytokines induced. However, the high level of inflammatory cytokines stimulated by Arcam particles suggests that the biological activity of particles with a size of approximately $60 \mu \mathrm{m}$ might be a result of other mechanisms that need further study.
Previously, we found that printing particles did not directly induce osteoclast maturation from precursors in the absence of RANKL (data not shown), which is consistent with a previous study. ${ }^{49}$ In this study, the promotion effect of particles on osteoclastogenesis was analyzed in the presence of M-CSF and RANKL. Although no statistically significant difference was observed in the effects of the printing particles, all of the particles at a non-cytotoxic concentration were found to effectively stimulate BMMs differentiation and promote the formation of multinucleated osteoclasts; moreover, the trend here was similar to that observed in vivo. These results indicate that bone loss induced by printing particles is also closely related to promotion of osteoclastogenesis.

However, some limitations exist in our current study. First, even though size-dependent effects of printing particles on osteolysis and the inflammatory reaction were clearly verified in this study, the underlying molecular mechanisms remain unclear and need to be investigated in future studies. Second, several typical printing particles from main supplier companies were chosen as study objects here. With the increased popularity of the $3 \mathrm{D}$ printing industry, more companies have been established in the last 3 years. Comprehensive investigations including distinct printing particle samples from other suppliers should be designed to better characterize the biological activity of printing particles. Despite these limitations, this study would still be valuable for improving the use of printing raw materials in the application of clinical implant fabrication.

\section{Conclusion}

In this study, we analyzed for the first time the in vitro and in vivo inflammatory reaction and osteolysis induced by commonly used Ti6Al4V printing particles and by one-time recycled particles; differences in particle size were observed not only between particles used for EBM and SLM techniques, but also between those from different companies based on the same technique. Recycled particles were found to possess a wider size distribution than the original particles. Although the effect was less serious than that observed with classic Ti particles, the printing particles stimulated macrophages to secrete proinflammatory cytokines and promoted BMMs differentiation into multinucleated osteoclasts in vitro, and triggered a proinflammatory reaction and osteolysis in vivo. At both low and high concentrations, particles sized approximately $20 \mu \mathrm{m}$ and $60 \mu \mathrm{m}$ strongly triggered proinflammatory reactions, osteoclastogenesis, and osteolysis. And recycled particles may have an even stronger effect than the original particles. The results of our findings might could serve as a valuable reference when using printing particles or examining the feasibility of recycling procedures for implant fabrication.

\section{Conflicts of interest}

There are no conflicts to declare. 


\section{Acknowledgements}

This investigation was supported by the National High Technology Research and Development Program of China (863 Program: 2015AA020308), Key Developing Disciplines Program of Shanghai Health System (2015ZB04904), Natural Science Foundation of Guangdong Province (2017A03031854), National Natural Science Foundation of China (81572156), and the Key Diseases and Technological Program of the Shanghai Health System (2013ZYJB0501).

\section{Notes and references}

1 S. L. Sing, J. An, W. Y. Yeong and F. E. Wiria, J. Orthop. Res., 2016, 34, 369-385.

2 A. Sidambe, Materials, 2014, 7, 8168.

3 P. Fischer, V. Romano, H. P. Weber, N. P. Karapatis, E. Boillat and R. Glardon, Acta Mater., 2003, 51, 1651-1662.

4 J. M. Williams, A. Adewunmi, R. M. Schek, C. L. Flanagan, P. H. Krebsbach, S. E. Feinberg, S. J. Hollister and S. Das, Biomaterials, 2005, 26, 4817-4827.

5 P. Heinl, L. Müller, C. Körner, R. F. Singer and F. A. Müller, Acta Biomater., 2008, 4, 1536-1544.

6 J. Parthasarathy, B. Starly, S. Raman and A. Christensen, J. Mech. Behav. Biomed. Mater., 2010, 3, 249-259.

7 G. Li, L. Wang, W. Pan, F. Yang, W. Jiang, X. Wu, X. Kong, K. Dai and Y. Hao, Sci. Rep., 2016, 6, 34072.

8 P. H. Warnke, T. Douglas, P. Wollny, E. Sherry, M. Steiner, S. Galonska, S. T. Becker, I. N. Springer, J. Wiltfang and S. Sivananthan, Tissue Eng., Part C, 2008, 15, 115-124.

9 A. Palmquist, A. Snis, L. Emanuelsson, M. Browne and P. Thomsen, J. Biomater. Appl., 2011, 27, 1003-1016.

10 H. Hasib, O. L. A. Harrysson and H. A. West, JOM, 2015, 67, 639-646.

11 C. M. Cheah, C. K. Chua, K. F. Leong and S. W. Chua, Int. J. Adv. Des. Manuf. Technol., 2003, 21, 291-301.

12 L. D. Dorr, K. R. Hilton, Z. Wan, G. D. Markovich and R. Bloebaum, Clin. Orthop. Relat. Res., 1996, 333, 108-117.

13 L. Saldaña and N. Vilaboa, Acta Biomater., 2010, 6, 16491660.

14 T. Akisue, T. W. Bauer, C. F. Farver and Y. Mochida, J. Biomed. Mater. Res., 2002, 59, 507-515.

15 C. Nich, J. Langlois, A. Marchadier, C. Vidal, M. Cohen-Solal, H. Petite and M. Hamadouche, Arthritis Res. Ther., 2011, 13, R100.

16 T.-h. Lin, Z. Yao, T. Sato, M. Keeney, C. Li, J. Pajarinen, F. Yang, K. Egashira and S. B. Goodman, Acta Biomater., 2014, 10, 3747-3755.

17 M. L. Wang, P. F. Sharkey and R. S. Tuan, J. Arthroplasty, 2004, 19, 1028-1038.

18 H. Gelb, H. Ralph Schumacher, J. Cuckler and D. G. Baker, J. Orthop. Res., 1994, 12, 83-92.

19 L. E. Murr, S. A. Quinones, S. M. Gaytan, M. I. Lopez, A. Rodela, E. Y. Martinez, D. H. Hernandez, E. Martinez, F. Medina and R. B. Wicker, J. Mech. Behav. Biomed. Mater., 2009, 2, 20-32.
20 H. Gong, K. Rafi, H. Gu, G. D. Janaki Ram, T. Starr and B. Stucker, Mater. Des., 2015, 86, 545-554.

21 H. Gong, K. Rafi, H. Gu, T. Starr and B. Stucker, Addit. Manuf., 2014, 1-4, 87-98.

22 C. Jiang, J. Shang, Z. Li, A. Qin, Z. Ouyang, X. Qu, H. Li, B. Tian, W. Wang, C. Wu, J. Wang and M. Dai, J. Cell. Physiol., 2016, 231, 142-151.

23 C. Jiang, F. Xiao, X. Gu, Z. Zhai, X. Liu, W. Wang, T. Tang, Y. Wang, Z. Zhu, K. Dai, A. Qin and J. Wang, Biochimie, 2015, 111, 107-118.

24 Z. Zhai, X. Qu, H. Li, K. Yang, P. Wan, L. Tan, Z. Ouyang, X. Liu, B. Tian, F. Xiao, W. Wang, C. Jiang, T. Tang, Q. Fan, A. Qin and K. Dai, Biomaterials, 2014, 35, 62996310.

25 H. Shao, J. Shen, M. Wang, J. Cui, Y. Wang, S. Zhu, W. Zhang, H. Yang, Y. Xu and D. Geng, Biomaterials, 2015, 60, 92-99.

26 T. Koga, M. Inui, K. Inoue, S. Kim, A. Suematsu, E. Kobayashi, T. Iwata, H. Ohnishi, T. Matozaki, T. Kodama, T. Taniguchi, H. Takayanagi and T. Takai, Nature, 2004, 428, 758-763.

27 J.-P. Hu, K. Nishishita, E. Sakai, H. Yoshida, Y. Kato, T. Tsukuba and K. Okamoto, Eur. J. Pharmacol., 2008, 580, 70-79.

28 B. Tian, T. Jiang, Z. Shao, Z. Zhai, H. Li, Q. Fan, X. Liu, Z. Ouyang, T. Tang, Q. Jiang, M. Zheng, K. Dai, A. Qin, Y. Yu and Z. Zhu, Biomaterials, 2014, 35, 8937-8950.

29 A. Sabokbar, R. Pandey and N. A. Athanasou, J. Mater. Sci.: Mater. Med., 2003, 14, 731-738.

30 M. Kolb, P. Bonniaud, T. Galt, P. J. Sime, M. M. Kelly, P. J. Margetts and J. Gauldie, Am. J. Respir. Cell Mol. Biol., 2002, 27, 141-150.

31 J. J. Jacobs, K. A. Roebuck, M. Archibeck, N. J. Hallab and T. T. Glant, Clin. Orthop. Relat. Res., 2001, 393, 71-77.

32 T. W. Bauer, Clin. Orthop. Relat. Res., 2002, 405, 138-143.

33 G. Vallés, P. González-Melendi, J. L. González-Carrasco, L. Saldaña, E. Sánchez-Sabaté, L. Munuera and N. Vilaboa, Biomaterials, 2006, 27, 5199-5211.

34 J. H. Dumbleton, M. T. Manley and A. A. Edidin, J. Arthroplasty, 2002, 17, 649-661.

35 C. A. Holding, D. M. Findlay, R. Stamenkov, S. D. Neale, H. Lucas, A. S. S. K. Dharmapatni, S. A. Callary, K. R. Shrestha, G. J. Atkins, D. W. Howie and D. R. Haynes, Biomaterials, 2006, 27, 5212-5219.

36 S. P. Nichols, A. Koh, W. L. Storm, J. H. Shin and M. H. Schoenfisch, Chem. Rev., 2013, 113, 2528-2549.

37 K. L. Helton, B. D. Ratner and N. A. Wisniewski, J. Diabetes Sci. Technol., 2011, 5, 632-646.

38 T. Lange, A. F. Schilling, F. Peters, J. Mujas, D. Wicklein and M. Amling, Biomaterials, 2011, 32, 4067-4075.

39 Y. Hirashima, N. Ishiguro, S. Kondo and H. Iwata, J. Biomed. Mater. Res., 2001, 56, 177-183.

40 S. D. Neale, Y. Fujikawa, A. Sabokbar, R. Gundle, D. W. Murray, S. E. Graves, D. W. Howie and N. A. Athanasou, J. Bone Jt. Surg., Br. Vol., 2000, 82, 892-900. 41 C. Jiang, Y. Zou, X. Liu, J. Shang, M. Cheng and M. Dai, J. Rare Earths, 2013, 31, 420-427. 
42 K. D. Merkel, J. M. Erdmann, K. P. McHugh, Y. Abu-Amer, F. P. Ross and S. L. Teitelbaum, Am. J. Pathol., 1999, 154, 203-210.

43 A. Sabokbar, R. Pandey and N. A. Athanasou, J. Mater. Sci.: Mater. Med., 2003, 14, 731-738.

44 T. W. Bauer and J. Schils, Skeletal Radiology, 1999, 28, 423432.

45 T. D. Zaveri, N. V. Dolgova, J. S. Lewis, K. Hamaker, M. J. Clare-Salzler and B. G. Keselowsky, Biomaterials, 2017, 115, 128-140.
46 K. D. Merkel, J. M. Erdmann, K. P. McHugh, Y. Abu-Amer, F. P. Ross and S. L. Teitelbaum, Am. J. Pathol., 1999, 154, 203-210.

47 J. Yadav, L. Samelko, P. Gilvar, K. McAllister and N. J. Hallab, Open Orthop. J., 2013, 7, 605-613.

48 N. J. Hallab and J. J. Jacobs, Front. Neuroendocrinol., 2017, 8, 5.

49 Y.-Y. Kong, H. Yoshida, I. Sarosi, H.-L. Tan, E. Timms, C. Capparelli, S. Morony, A. J. Oliveira-dos-Santos, G. Van, A. Itie, W. Khoo, A. Wakeham, C. R. Dunstan, D. L. Lacey, T. W. Mak, W. J. Boyle and J. M. Penninger, Nature, 1999, 397, 315-323. 